\title{
High Quality InAs Grown on Silicon Substrate
}

Jinan Liu

Northwestern Polytechnical University

127 West Youyi Road, Beilin District, Xi'an Shaanxi, 710072, P.R.China.

Email: 1jnan202015@outlook.com

We have investigated growth of InAs on Si substrates by metal organic chemical vapor deposition. The main investigation is the effect of incorporating more nucleation layers into the growth process. The addition of more nucleation layers clearly correlates with the surface morphology and quality of the InAs layer. Morphological and structural characterizations prove that a perfect quality InAs layer is achieved by incorporating five nucleation layers.

\section{Introduction:}

InAs is an attractive material for optical and high-speed devices due to its high electron mobility and narrow direct band gap [1,2]. However, integration of InAs on Si has remained a challenge over the last decades [3,4]. The large lattice mismatch of between InAs and Si substrates is about $11.6 \%$ which creates dislocations and strain for further growth of epitaxial layers. Another challenge is the different thermal expansion coefficients of InAs and Si [5,6]. In this paper, we report five-steps growth of InAs on Si by optimizing the growth conditions and by introducing totally 5 five nucleation layers into the growth process. Firstly, a nucleation layer is grown at low temperature and high V/III ratio with a thickness of $10 \mathrm{~nm}$. Then, a second layer growth is followed at the same high temperature for $55 \mathrm{~min}$ with a low V/III ratio. Figure 1a shows a schematic structure of the InAs layer with 5 nucleation layers.
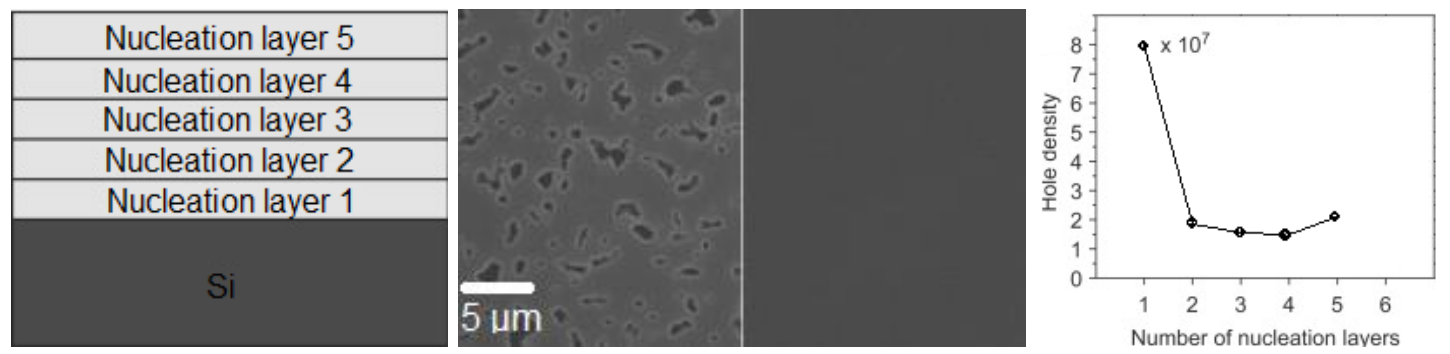

Fig. 1 (a) Schematic structure of multi nucleation layer to obtain InAs. (b) SEM image of InAs with one (lelf) and 5 (right) nucleation layers and (c) Hole density with multi nucleation layers.

Experimental:

Highly resistive $\mathrm{Si}$ was used as substrates. Growth was performed in a horizontal MOVPE reactor. Trimethylindium (TMIn) and Arsine $\left(\mathrm{AsH}_{3}\right)$ were used as precursors with hydrogen as a carrier gas with a total flow of $12 \mathrm{l} / \mathrm{min}$ and a reactor pressure of $100 \mathrm{mbar}$. The growth of the nucleation layer was performed at a low temperature of $350^{\circ} \mathrm{C}$ for $10 \mathrm{~min}$ followed by ramping up to $600^{\circ} \mathrm{C}$, where the nucleation layer was annealed for $6 \mathrm{~min}$ and a second layer growth continued at the same high temperature. The TMIn molar fraction was constant during the deposition at $2 \times 10^{-5}$. The $\mathrm{AsH}_{3}$ molar fraction was $3 \times 10^{-3}$ during the growth of the nucleation layer and was decreased one order of magnitude for the growth of the second layer. For heteroepitaxial growth of $\mathrm{GaSb}$ on InAs, the TEGa molar fraction was set to $2 \times 10^{-4}$ and the TMSb molar fraction was varied from $2 \times 10^{-4}$ to $3 \times 10^{-4}$. The GaSb layers were grown at the temperature of $650^{\circ} \mathrm{C}$ for $10 \mathrm{~min}$. The surface morphology of the InAs layer was evaluated by scanning electron microscopy (SEM), 
atomic force microscopy (AFM) was used to determine the atomic roughness and dislocation structure of our samples. The quality, composition, and strain of the samples were determined by high resolution X-ray diffraction (XRD) measurement.

\section{Result:}

Deposition of InAs on Si using one nucleation step results in layers with holes remaining on the surface [7]. To investigate the effect of incorporating more nucleation layers, we have grown a set of 5 samples with one to 5 nucleation layers. Inserting additional nucleation layers results in a thickness variation from 200 to $300 \mathrm{~nm}$. SEM inspection of the samples clearly indicates that there is a major improvement in the surface morphology and reduction in holes as we increase from one (Fig. 1b) to two nucleation layers. From the four nucleation layered samples, the hole formation is suppressed down to $5 \times 10^{6} \mathrm{~cm}^{-2}$ (Fig. 1c). For the cases of five and 5 nucleation layers, the surface structure looks the same as the four nucleation layered sample (Fig. 1c). The density of holes on the surface decreases significantly with incorporating more nucleation layers.

We have analyzed the InAs surface morphology by AFM characterization. We observed that surface roughness (RMS) of a sample with one nucleation layer in flat region is $2.1 \mathrm{~nm}$. The RMS value decreases to $1.5 \mathrm{~nm}$ for the sample with 2 nucleation layers. Starting from three nucleation layers, the RMS value is reduced to $0.8 \mathrm{~nm}$. Fig. 2a shows a derivative of an AFM image obtained for the 5 nucleation layered sample. As shown in Fig. 2a, steps are flowing downward relative to the AFM image and step height corresponds to InAs lattice constant height [8]. It should be mentioned that there are a few reports on step-flow growth of InAs on GaAs which has a lattice mismatch of $\sim 7 \%[9,10]$. However, we are unaware of any experimental result on successful step-flow growth of InAs on $\mathrm{Si}(111)$, which has a larger lattice mismatch $(11.6 \%)$. We believe that this is the first comprehensive report and the result of this study could be applied in a variety of device applications.
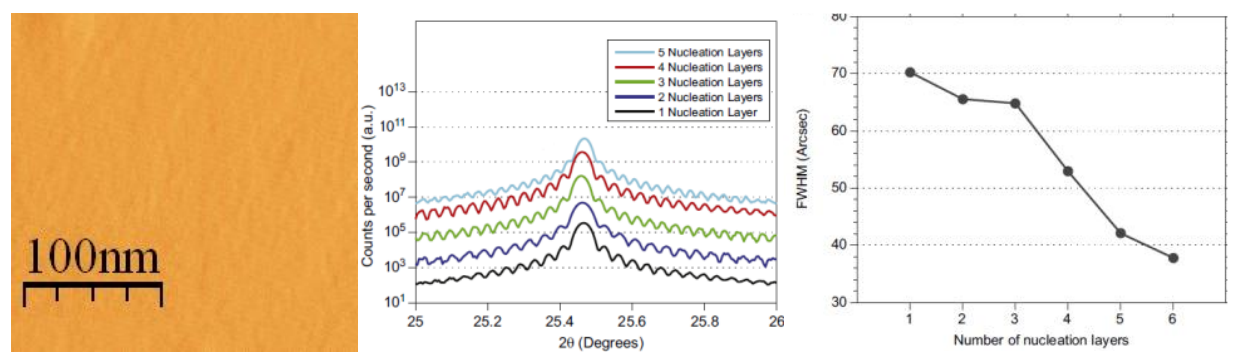

Fig. 2 (a) 250x250nm AFM image of 5 nucleation layers shows high surface roughness. (b) FWHM versus number of nucleation layers and (c) XRD result of all samples.

XRD characterization of the samples demonstrates the high quality of the InAs layer by

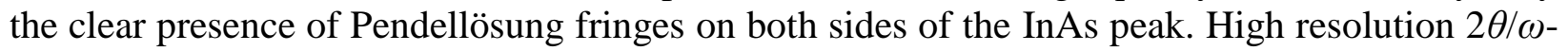
scans of six samples with one-5 nucleation layers are shown in Fig. $2 b$. The XRD data shows that as the number of nucleation layers increases, more Pendellösung fringes on either side of the InAs peak are distinguished and the spacing between the fringes decreases. This indicates that the introduction of more nucleation layers results in an improvement in the film quality. FWHM values from the InAs rocking curves are shown in Fig. 2c and it clearly shows a decrease in the FWHM data as more nucleation layers are incorporated and the thickness increases. This decrease of 
FWHM values could be related to an improvement in film quality and a reduction of the dislocation density.

Conclusion:

This paper reports on successful growth of InAs thin layers on Si (111) substrates. We have investigated the influence of incorporating additional nucleation layers on the surface morphology and film quality. We have characterized different samples with one-5 nucleation layers. It is observed that by inserting additional nucleation layers in the growth process, the surface morphology and quality of the InAs improve significantly. Our systematic study revealed that using four nucleation layers results in the formation of high quality InAs thin layers. This technology demonstrates the possibility to integrate high performance on Si substrate.

\section{References}

[1] H. D. Trinh et al., IEEE Electron Device Letters, vol. 32, No. 6, June 2011

DOI: $10.1109 /$ LED.2011.2128853

[2] Hai-Dang Trinh et al., Japanese Journal of Applied Physics 49 (2010) 111201

https://doi.org/10.1143/JJAP.49.111201

[3] Kartika Chandra Sahoo et al., Japanese Journal of Applied Physics 48 (2009) 126508

https://doi.org/10.1143/JJAP.48.126508

[4] Chen-Chen Chung et al., Nanoscale Research Letters 2014, 9:338

https://doi.org/10.1186/1556-276X-9-338

[5] Chen-Chen Chung et al., Electron. Mater. Lett., Vol. 10, No. 2 (2014), pp. 457-460

https://doi.org/10.1007/s13391-013-3202-3

[6] Hong-Quan Nguyen et al., Applied Physics Express 5 (2012) 055503

https://doi.org/10.1143/APEX.5.055503

[7] Chen-Chen Chung et al., Mater. Res. Express 2 (2015) 055505

https://doi.org/10.1088/2053-1591/2/5/055505

[8] Hung Wei Yu et al., Japanese Journal of Applied Physics 51 (2012) 080208

http://dx.doi.org/10.1143/JJAP.51.080208

[9] Chi-Lang Nguyen et al., Electron. Mater. Lett., Vol. 10, No. 4 (2014), pp. 759-762

https://doi.org/10.1007/s13391-014-4016-7

[10] Chen-Chen Chung et al., ECS Trans. 2013 volume 50, issue 48, 5-8

DOI: $\underline{10.1149 / 05048.0005 e c s t}$ 